\title{
Electron Cryotomography Reveals That The Caulobacter crescentus Cytoskeleton Is Surprisingly Complex
}

\author{
D. P. Dias*, A. Briegel*, R. B. Jensen**, A. Frangakis***, and G. J. Jensen* \\ *Div. of Biology, California Inst. of Technology, 1200 E. California Blvd, Pasadena CA 91125 \\ **Dept. of Life Sciences and Chemistry, Roskilde University, Universitetsvej 1, Postbox 260, DK- \\ 4000 Roskilde, Denmark \\ ***Computational and Structural Biology, European Molecular Biology Lab, Heidelberg, Germany
}

Fluorescence light microscopy of bacteria has shown that several prokaryotic proteins localize in extended, filament-like patterns in vivo [1]. Some, including FtsZ, MreB, and CreS, have further been shown by electron microscopy to form actual filaments in vitro [2,3]. Despite the critical roles these proteins play in cell shape, genome segregation, and cell division, molecular mechanisms have remained obscure in part because no corresponding filaments have ever been seen inside cells by traditional electron microscopy.

We used both single- and dual-axis electron cryotomography to image wild-type Caulobacter crescentus in amorphous ice, which preserves the cells in a near-native state [4]. We obtained over 50 three-dimensional reconstructions of whole cells with unprecedented clarity and fidelity. The reconstruction in Fig. 1 is typical of our results, in which the surface layer, the inner and outer membranes, and even the peptidoglycan layer between the membranes are all clearly visible. The length of each cell and the occasional presence of flagella, pili, stalks, and mid-cell constrictions were used as landmarks of each cell's progress through the cell cycle.

However, the most striking feature of the cell shown in Figure 1 was the bundle of filaments lying just inside the inner membrane on the concave side of the cell at approximately its midpoint. Throughout the many reconstructions we obtained, a total of five distinct types of cytoskeletal filament bundles were discovered, including two that appeared to be consistent with previous light microscopy results in $C$. crescentus and three types that were novel. The filament in Figure 1 is of the first type, which is similar in position to the light microscopy results for the CreS protein. The second type of filament had a position similar to that expected for FtsZ or MreB during cell division. One of the novel patterns consisted of pairs of long filaments extending from the cell poles through the middle of the cytoplasm, as shown in Figure 2. These results are intriguing because this pattern is reminiscent of the fluorescence light microscopy results for ParM, which segregates the R1 plasmid in E. coli before cell division [5].

We in the process of building on these results by imaging various $C$. crescentus strains with mutations in cytoskeletal filament-forming genes to investigate the identity of the filaments seen in the wild-type cells.

\section{References}

[1] L. A. Amos, et al., Curr. Op. Cell Biol. 16 (2004) 24-31.

[2] N. Ausmees, et al. Cell 115 (2003) 705-713.

[3] J. Lowe, et al. Ann. Rev. Biophys. Biomol. Struct. 33 (2004) 177-198.

[4] C. Iancu, et al. J. Struct. Biol. 151 (2005) 577-588.

[5] J. Moeller-Jensen, et al. Mol. Cell 12 (2004) 1243-1250. 


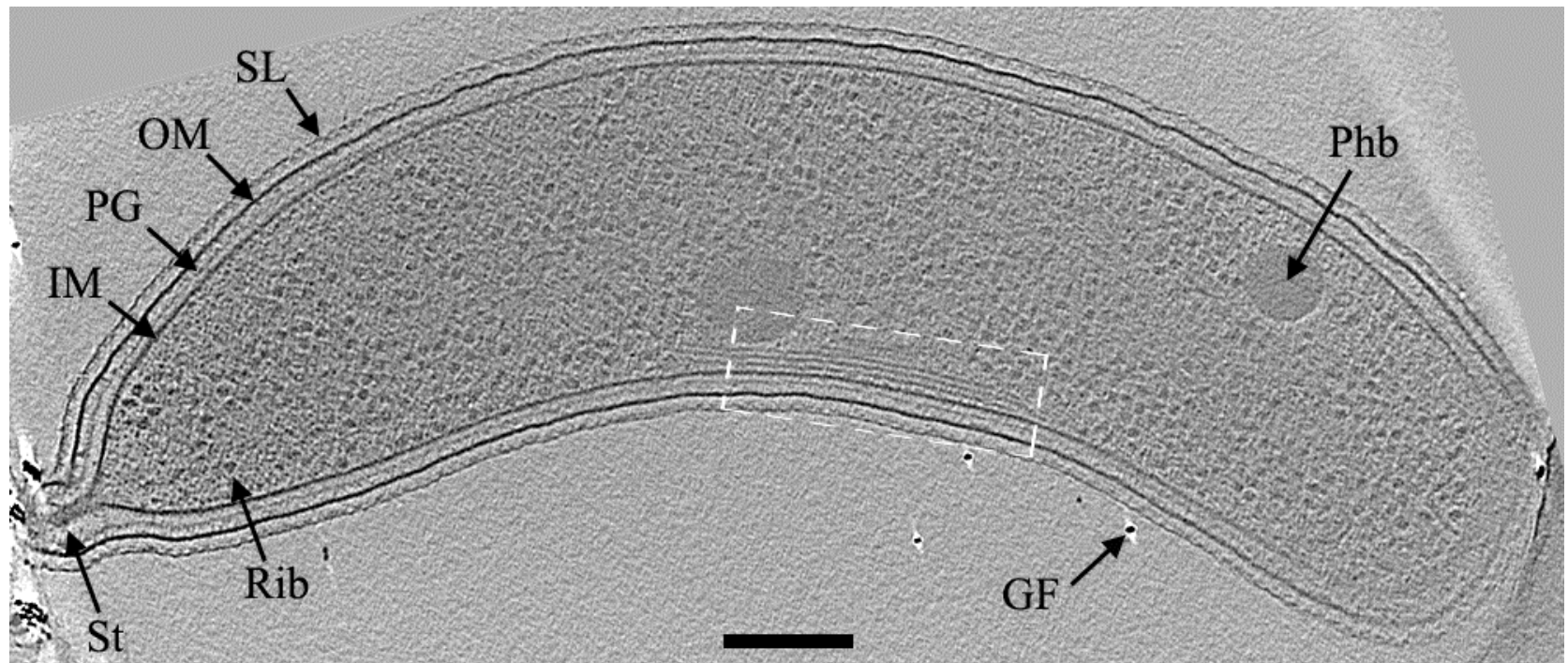

Figure 1. Slice through the three-dimensional reconstruction of a representative $C$. crescentus cell : SL_-surface layer; OM — outer membrane; PG_-peptidoglycan layer; IM — inner membrane; St stalk; Rib_ribosome; GF_-gold fiducial; Phb—poly- $\beta$-hydroxybutyrate granule. Scale bar 200 $\mathrm{nm}$.

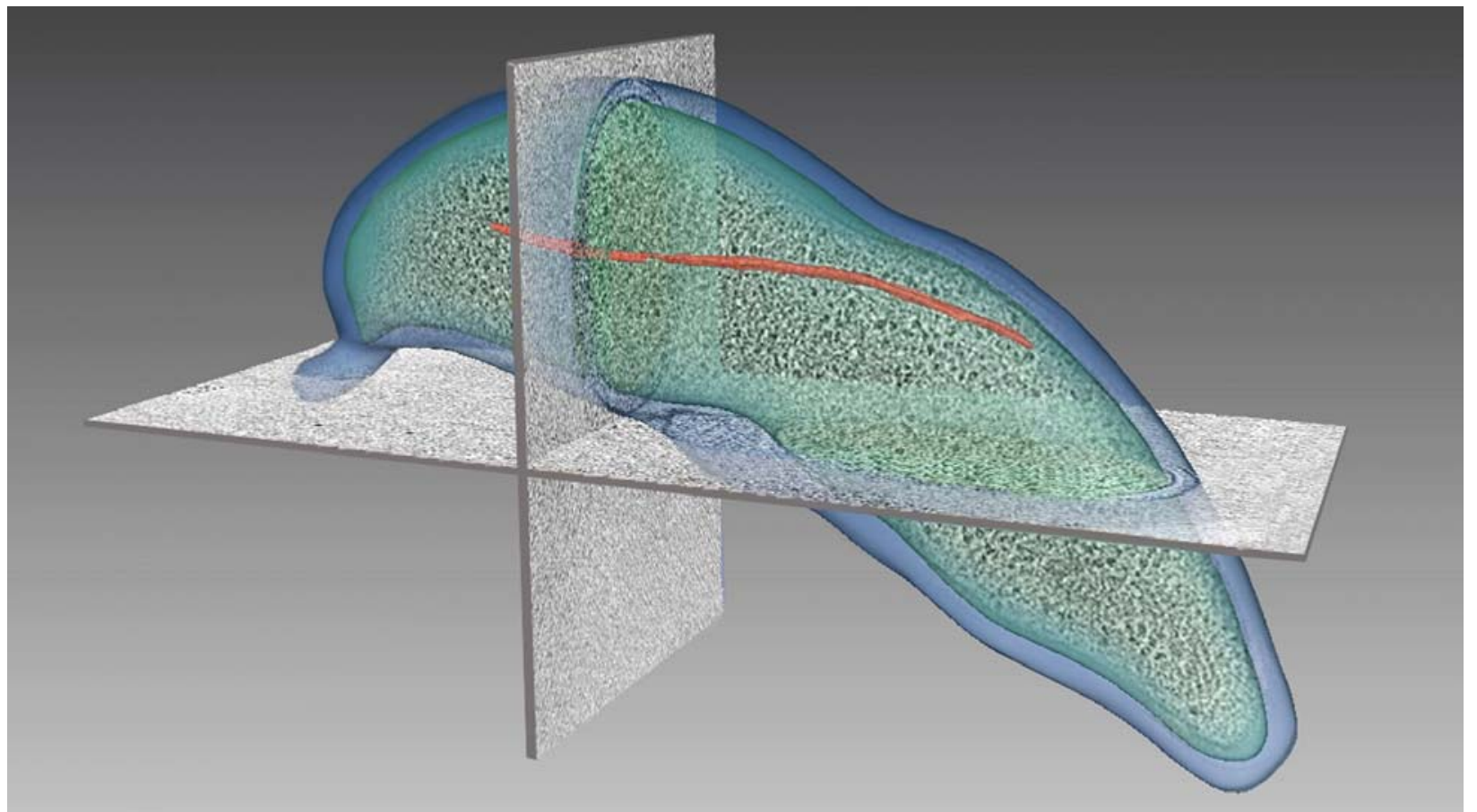

Figure 2. Three-dimensional rendition of a whole cell, showing the full extent of the novel filament pattern (red). 